\title{
Two-stage stochastic programming approach for limited medical reserves allocation under uncertainties
}

\author{
Yuwei Zhang ${ }^{1,2} \cdot$ Zhenping $\mathrm{Li}^{2} \cdot$ Pengbo $\mathrm{Jiao}^{2} \cdot$ Shen $\mathrm{Zhu}^{2}$
}

Received: 17 April 2021 / Accepted: 3 August 2021 / Published online: 12 August 2021

(c) The Author(s) 2021

\begin{abstract}
At the early stage of public health emergencies, when the conventional medical reserves prepared are insufficient, and productivity could temporarily not meet the surge in demand, donations can be used to cover excess demand for medical supplies to a large extent. This paper explicitly considers the allocation problem of limited medical reserves during a public health emergency, incorporating uncertainty in demand and donated supplies and the priorities of health care centers. The problem is formulated as a two-stage stochastic program that regards the donated supplies as an efficient recourse action, aiming to minimize the total losses. The optimal allocation strategy of limited medical reserves and donations is obtained by solving the model using Gurobi solver. Finally, the effectiveness of the proposed approach is verified by a series of computational results, which show that the solutions of our method not only benefit the emergency demand fulfill rate but reduce the total losses as well.
\end{abstract}

Keywords Limited medical reserves allocation $\cdot$ Donations $\cdot$ Uncertain demand $\cdot$ Priority $\cdot$ Two-stage stochastic programming

\section{Introduction}

The storage pool consists of a fixed quantity of medical reserves prepared for emergencies, including public health emergencies and natural disasters such as an epidemic [1, 2], earthquake and flood [3-5]. Medical reserves are set for emergencies that occur occasionally and usually result in uncertain demand. A very high minimum stock level to ensure sufficient supplies will result in excessive space because of low mobility, as well as substantial waste due to expiration. Therefore, medical reserves in the storage pool for emergency supplies maintain a relatively low (limited) stock level [6]. This work explicitly focuses on how to allocate limited medical reserves when a public health emergency occurs. In the early stage of a large-scale public health emergency, it takes time to organize manufacturers

Zhenping Li

lizhenping@bwu.edu.cn

1 School of Management and Engineering, Capital University of Economics and Business, 121 Zhangjialukou, Huaxiang Fengtai District, Beijing 100070, Republic of China

2 School of Information, Beijing Wuzi University, 1 Fuhe Street, Tongzhou District, Beijing 101149, Republic of China to restore production to step up supplies, especially during unusual times. Consequently, productivity may temporarily not meet the surge in demand arising from emergencies. As medical reserves prepared for an emergency response might be insufficient, donated supplies can be served as a recourse action to cover some excess demand to a large extent.

Certainly, how to utilize scarce medical materials prepared for emergency response to maximize their value is a vital issue. In the aftermath of a large-scale public health emergency, to reduce mortality and raise admission and cure rates, health care centers are grouped into two classes, designated hospitals and general hospitals. Designated hospitals mainly receive and cure patients in severe conditions, while general hospitals converted from large buildings are for mild cases, suspected cases, and close contacts. That severe patients obtain medical treatment plays a critical role in preventing and controlling a large-scale public health emergency, indicating that the same reserves distributed to the designated hospital will yield more utility than general hospitals. Therefore, when the pre-positioned medical reserves are inadequate, the designated hospital for severe patients should be prioritized to maximize the value of limited reserves. Inspired by Shin and Lee [7], we introduce priority to describe the weight of importance. In addition, health

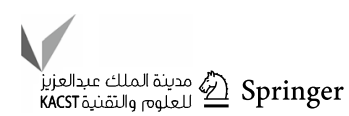


care centers' requirements for resources and donated supplies cause considerable variability in the decision-making process. We incorporate and analyze many important reallife issues: limited medical reserves, the priorities of health care centers, uncertainty in demand and donated supplies. This decision process is complex and involves the allocation of multiple supplies, which has a significant impact on both the quality and cost of service.

The rest of the paper is organized as follows: "Literature review" reviews the relevant literature in the area of the medical resources allocation. "Problem formulation" delineates the problem, notations, and the quantitative model addressed in this study. A modeling framework for the allocation of limited medical reserves is introduced, and it can be formulated as a two-stage stochastic program that involves uncertainty in demand and donations. "Computational experiments" reports computational experiment results performed by Gurobi for solving the model proposed in "Problem formulation". The paper ends with overall conclusions and some future research directions.

\section{Literature review}

The medical resources allocation problem has been studied in several recent studies, and it has considerable potential in the management of medical resources from emergency scenarios.

Our work is closely related to the resource allocation problem, which has attracted much attention because of its practical relevance. There are increasing and various studies concentrating on the medical resource allocation problem. Cao et al. [8] proposed a discrete event simulation model to determine the most reasonable scarce medical resources allocation principle from four principles and evaluated their effects by considering the trade-off between survival efficiency and moral issues in the emergency response. Su et al. [9] defined disaster response alliance as a form of teamwork and proposed a multi-constrained integer linear programming model, which was solved by an algorithm based on differential evolution and heuristic exploration to allocate multiple emergency resources to various events in a parallel way. To handle the problem of multi-resource and multi-location based on capability, Altay [10] established an integer programming model to allocate resources better. Yu et al. [11] paid more attention to resource allocation's efficiency, effectiveness and fairness. Two equivalent models were applied to address this problem which was discussed well through a rollout algorithm. Yu et al. [12] applied an improved dynamic programming method to measure critical resource allocation effectiveness and fairness.

There are several studies considering limited medical resources in the resource allocation problem. Yu et al. [13] developed a Timed Colored Petri net to describe and model multi-resource-multi-activity. The hospital's limited medical resources can be used more reasonably through the medical information integration system, and the activities become more efficient. Ho et al. [14] considered dynamic resource allocation and optimized sequential intervention policies under resource constraints with a multi-fidelity optimization model and method. In public healthcare, Lai et al. [15] modeled resource allocation by a team-DEA analysis which could be used to optimally allocate resources.

Several studies involve uncertain conditions. A possibilistic programming model was presented by Kargar et al. [16] for a liver transportation and allocation problem, which takes uncertainty into account and trade-off between quality indicators (i.e., efficiency and fairness). Their models have maximized the patients' survival probability and minimized transportation cost and time. Bastian et al. [17] presented a stochastic multi-objective automatic optimization model for allocating medical resources in fixed-input health systems. The model automatically determined the hospital level to reallocate the system's input resources for the optimization of the overall system performance. Huang et al. [18] studied the dynamic allocation and scheduling of stochastic medical resources in surgery. They analyzed the bottleneck resources' problem for optimizing medical resource scheduling through the drum-buffer-rope scheduling manner. To address the challenge of medical staff allocation and the scheduling in random environments, a two-stage algorithm based on goal planning has been proposed by Chen et al. [19] to identify the minimum number of medical staff needed and arrange the optimal schedule for them. Zonouzi and Kargari [20] constructed a new mathematical model based on the data mining methods to maximize ambulances' coverage to demand points under uncertain conditions of rescue stations. The data mining methods were also used to estimate allocation problems under uncertainty, Franco and Alfonso [21] used a random counterpart optimization approach and proposed two models considering different uncertainty sources. Zhang [22] studied a general resource planning and allocation problem under uncertain environment by two-stage stochastic programming.

$\mathrm{Hu}$ et al. [23] provided a bi-objective robust resource allocation model to maximize emergency resources' efficiency and fairness, considering scarce supplies and many types of uncertainty. Boostani et al. [24] investigated and simulated the uncertainty in disaster relief conditions by a multiobjective mixed-integer stochastic programming model. Yang et al. [25] formed a distributed robust optimization model for emergency supplies pre-positioning under high uncertainty and limited information. Gao [26] designed a multi-commodity rebalancing problem through a bi-level stochastic mixed-integer programming model. Zhang et al. [27] established a two-stage stochastic programming model 
and used the Lagrangian relaxation method to deal with mobile blood collecting facilities' location and allocation. Xiang et al. [28] formulated a two-objective robust model to study the berth allocation problem when considering uncertain factors.

Some researches specifically focus on the allocation under the demand uncertainty. To determine optimal policies for medical drug distribution, Nikzad et al. [29] used a twostage stochastic programming method to incorporate demand uncertainty into the optimization model and evaluated the effectiveness of the model by two different approaches based on chance constraints. Batista et al. [30] studied the tradeoff between the allocation of medical resources and the cost of service by a bi-objective stochastic programming model while considering the uncertainty in demand and capacity.

Furthermore, the problem of limited resource allocation in uncertain conditions has also been carefully explored by scholars. Feng et al. [31] constructed a stochastic bi-objective mathematical model in emergency departments, while their studies developed a multi-objective simulation method to deal with the difficulties in the multi-objective limited medical resources allocation. Tang and Wang [32] used an adjustable robust optimization model to explore surgical capacity allocation with uncertain demand and reduce the losses of income due to resource shortages.

In general, the works of literature above have conducted various researches on the allocation of medical resources. Nevertheless, most of the existing works assume that either medical resources for emergencies are sufficient so that demand arising from emergencies can be satisfied or the allocation is implemented, while several critical conditions are known, such as demand or supply. We observe that both uncertainties in demand and limitation of supplies are hardly avoidable, since emergencies are sudden and unpredictable, demand is not accurately known, and resources are not always sufficient.

Donated supplies can be used to cover some excess demand to a large extent when medical reserves prepared for emergency response are insufficient. Therefore, it is necessary to take donated supplies into account. In this research, we discuss donated supplies, limited medical reserves, and uncertainty in demand. This is an exploratory study on incorporating donated supplies in a limited resources allocation problem.

\section{Problem formulation}

\section{Problem description}

We introduce priority which refers to a weight of importance. As medical reserves prepared for emergency response are insufficient, health care centers' demand with high priority, such as designated hospitals for severe patients, should be prioritized.

When both the demand of health care centers and donated supplies are uncertain, the allocation of limited medical reserves for each health care center should be performed. Since productivity may temporarily not meet the surge in demand caused by the emergencies at early stages, donations can be regarded as an efficient recourse action to cover some demand which medical reserves fail to satisfy, while the demand and donations become known.

There are several assumptions itemized:

(1) When public health emergencies occur, the control and management of medical materials are performed by the government. Since the cost related to transportation distance is not included, the total of medical reserves pre-positioned in multiple locations (even the appropriate medical storage of each hospital) can be regarded as the inventory level stored in one storage pool. The government undertakes the task of distribution.

(2) The probability distribution of the random variables is known and derived from historical data.

(3) The lower and higher demand level based on a specific scenario is employed to describe each health care center's demand fluctuation. The number of supplies received by health care centers that is more than the higher demand level or less than the lower demand level will generate related costs, i.e., penalty cost and shortage cost.

(4) Donated supplies are all distributed by charitable organizations without storage in the storage pool.

(5) Inventory level of a single type of product in the storage pool for emergency supplies, that is, the amount of medical reserves is known.

The decisions to be made are:

(1) how to allocate limited medical reserves in the storage pool;

(2) how to allocate uncertain donated supplies.

The objective is to minimize the total cost, which has several terms:

(1) the allocation costs involving distribution cost and labor cost;

(2) recourse cost, i.e., to qualify "losses" related to the allocation of limited medical reserves, and other costs are incurred after the first-stage decision is made due to secondary allocation, shortage, and surplus. 


\section{A two-stage stochastic programming formulation}

In this section, we propose a two-stage stochastic optimization model for the allocation problem of limited medical reserves. Uncertainty in demand and donations is taken into consideration. The problem has a two-stage characteristic, i.e., before the realization of uncertainty, there is a hereand-now decision to make. After the realization of uncertainty, recourse actions are possible. Before presenting the model, we introduce some notations used for the sets, indices, parameters, and decision variables of the formulation.

\section{(1) Notation}

\section{Sets}

I: set of health care centers;

$S$ : set of possible scenarios;

\section{Indices}

$i$ : index of health care center;

$s$ : index of scenario;

\section{Parameters}

$r$ : inventory level of medical reserves in the storage pool;

$a_{i}$ : unit cost for allocating the medical reserves to health care center $i$

$q_{i}$ : unit shortage cost for unsatisfied demand (less than the lower demand level) of health care center $i$;

$c_{i}$ : unit penalty cost for surplus supplies received by health care center $i$;

$k_{i}$ : unit cost for allocating the donated supplies to health care center $i$;

$v_{i}$ : priority of health care center $i$;

\section{Stochastic symbols}

$\Omega=\left\{\omega_{1}, \omega_{2}, \ldots, \omega_{|s|}\right\}$ : set of all realizations of scenarios $\omega$, where $\omega \in \Omega$ represents a random event, i.e., a public health emergency, which influences several random variables; $d_{i}(\omega)$ : lower demand level of health care center $i$ under scenario $\omega$;

$h_{i}(\omega)$ : higher demand level of health care center $i$ under scenario $\omega$;

$e(\omega)$ : maximum available donated supplies under scenario $\omega$

$\xi(\omega)$ : vector of the random variables under scenario $\omega$;

Note that the second-stage decision variables are typically not the same under different realizations of $\omega$. Piecing together the stochastic components of second-stage data, we obtain a vector $\xi(\omega)=\left(d_{i}(\omega), h_{i}(\omega), e(\omega)\right)$. A single random event $\omega$ influences all components of $\xi$.

Decision variables

$x_{i}$ : amount of medical reserves allocated to health care center $i$;

$y_{i}(x, \xi)$ : amount of donations allocated to health care center $i$ when $\xi$ is realized; $b_{i}(x, \xi)$ : amount of surplus of health care center $i$ when $\xi$ is realized;

$\phi_{i}(x, \xi)$ : amount of shortage of health care center $i$ when $\xi$ is realized.

\section{(2) A two-stage stochastic program}

In our modeling framework, decisions are made in two stages. The first-stage variables are here-and-now decisions represented by the $x_{i}$. These variables decide on allocating reserves in the storage pool for emergency medical supplies, which are taken before demand and donations are known. The second-stage decisions are taken after the realization of uncertainty, which can be regarded as recourse actions. In this problem, we have three possible such actions: allocation of donations, surplus and shortage of medical supplies. Note that the second-stage variables depend on the first-stage decision variables as well as the realization of the random variables denoted by the vector $\xi(\omega)$, whose realizations correspond to the various scenarios $\omega \in \Omega$. For simplicity, we use $\xi$ to replace $\xi(\omega)$. Accordingly, we denote the second-stage variables or recourse decisions as $y_{i}(x, \xi), b_{i}(x, \xi)$, and $\phi_{i}(x, \xi)$, respectively. When the random event $\omega$ occurs, the second-stage parameters $d_{i}(\omega), h_{i}(\omega), e(\omega)$, become known. Then, the second-stage variables $y_{i}(x, \xi), b_{i}(x, \xi)$, and $\phi_{i}(x, \xi)$ must be taken. The recourse decisions thus involve the allocation of medical reserves.

The first-stage problem:

$\operatorname{minimize} \sum_{i \in I} a_{i} x_{i}+E_{\xi} Q(x, \xi)$

subject to $\sum_{i \in I} x_{i} \leq r$

$x_{i} \geq 0 \quad \forall i \in I$

The second-stage problem:

$Q(x, \xi)=\min \sum_{i \in I} v_{i} q_{i} \phi_{i}(x, \xi)+\sum_{i \in I} c_{i} b_{i}(x, \xi)+\sum_{i \in I} k_{i} y_{i}(x, \xi)$

subject to $x_{i}+y_{i}(x, \xi)+\phi_{i}(x, \xi) \geq d_{i}(\omega) \quad \forall i \in I$

$x_{i}+y_{i}(x, \xi)-b_{i}(x, \xi) \leq h_{i}(\omega) \quad \forall i \in I$

$\sum_{i \in I} y_{i}(x, \xi) \leq e(\omega)$

$y_{i}(x, \xi) \geq 0 \forall i \in I$

$\phi_{i}(x, \xi) \geq 0 \quad \forall i \in I$ 
$b_{i}(x, \xi) \geq 0 \quad \forall i \in I$

The first-stage problem defines the allocation strategies of medical reserves for health care centers before demand and donations are known. The objective function (1) is to minimize the total cost, which consists of cost for allocation of medical reserves and recourse cost, where $E_{\xi} Q(x, \xi)$ denotes the expected value of the second-stage problem; constraints (2) imply the total allocation of medical reserves must be less than or equal to the inventory level; constraints (3) represent that the first-stage variables are non-negative.

Note that in the second-stage problem, decision variables $x_{i}$ are fixed. After demand and donations are known, the allocation strategy of donated supplies for health care centers will be further made, and it is useful for supporting decisions on the first stage. The second-stage objective is to minimize the recourse cost related to the first-stage decision, which involves other costs incurred due to the allocation of donated supplies, shortage, and surplus.

Constraints (5) and (6) describe the relationship between the demand level and the total allocation, consisting of both medical reserves and donated supplies. Constraints (7) ensure the amount of donations allocation is no more than the maximum available donated supplies when scenario $\omega$ realized. Constraints (8), (9), and (10) state that the secondstage variables are non-negative.

\section{Extensive form of linear equivalent}

When the realizations of the scenario $\omega \in \Omega$ are finite, i.e., $\Omega$ contains a finite number of elements, the second-stage decision variables can be indexed and the two-stage stochastic program mentioned above can be formulated as an equivalent linear programming model. Uncertainties can be represented as a finite set of scenarios, which is denoted as $S$. Each scenario occurs with a known probability $p_{s}$, where $\sum_{s \in S} p_{s}=1$. We redefine the second-stage decision variables and parameters concerning demand level and donations as follows:

$d_{i s}$ : lower demand level of health care center $i$ when scenario $s$ occurs;

$h_{i s}$ : higher demand level of health care center $i$ when scenario $s$ occurs;

$e_{s}$ : maximum available donated supplies when scenario $s$ occurs;

$y_{i s}$ : amount of donations allocated to health care center $i$ when scenario $s$ occurs;

$b_{i s}$ : amount of surplus of health care center $i$ when scenario $s$ occurs;

$\phi_{i s}:$ amount of shortage of health care center $i$ when scenario $s$ occurs;
The extensive form of the equivalent linear programming model is formulated as follows:

$$
\begin{aligned}
& \min \sum_{i \in I} a x+\sum_{s \in S} p_{s}\left(\sum_{i \in I} v_{i} q_{i} \phi_{i}+\sum_{i \in I} c_{i} b_{i}+\sum_{i \in I} k_{i} y_{i}\right) \\
& \sum_{i \in I} x_{i} \leq r
\end{aligned}
$$

$x_{i}+y_{i s}+\phi_{i s} \geq d_{i s} \quad \forall i \in I, s \in S$

$x_{i}+y_{i s}-b_{i s} \leq h_{i s} \quad \forall i \in I, s \in S$

$\sum_{i \in I} y_{i s} \leq e_{s} \quad s \in S$

$x_{i s} \geq 0 \quad \forall i \in I, s \in S$

$y_{i s} \geq 0 \quad \forall i \in I, s \in S$

$\phi_{i s} \geq 0 \quad \forall i \in I, s \in S$

$b_{i s} \geq 0 \quad \forall i \in I, s \in S$

Note that the formulation above can be solved by commercial solvers directly, such as Gurobi.

Proposition 1 The constraint matrix of the equivalent linear programming model is totally unimodular (details of the proof are provided in Appendix).

According to Proposition 1, we can directly solve the linear equivalent to obtain the optimal solution of the corresponding integer programming model, i.e., the linear program has an optimal integral solution. Therefore, the proposed approach can be applied to large-scale integer programming of the limited medical reserves allocation problems.

\section{Expected value problem and wait-and-see problem}

The expected value problem replaces all random variables with their expected values to mitigate uncertainty. In our case, replacing the quantity of demand and donations with their expected values leads to a deterministic problem. The expected result of using the solution of the expected value problem is defined as $E E V$. The gap between $E E V$ and the optimal value of a stochastic program $(S P)$ is defined as the value of the stochastic solution or VSS [33].

The expected value of perfect information or EVPI measures the maximal amount the decision-maker would be ready 
to pay to get the information in advance. To obtain EVPI, the optimization problem associated with each scenario $\omega$, i.e., distribution problem, should be solved first. When the demand level of each health care center and donations are known before allocating the medical reserves, the decision process corresponds to the wait-and-see approach. Find the optimal objective values of the problem for all scenarios and compute the expected value, which is commonly denoted by WS. EVPI is the gap between the optimal value of a stochastic program $(S P)$ and WS [33].

VSS and EVPI are two measures of uncertainty effects. We introduce alternative measures, $V S S_{\text {gap }}$ and $E V P I_{\text {gap }}$, which are independent from the magnitude of the values involved. $V S S_{\text {gap }}$ and $E V P I_{\text {gap }}$ are calculated as follows:

$V S S_{\text {gap }}=(E E V-S P) / S P$

$E V P I_{\text {gap }}=(S P-W S) / W S$

\section{Computational experiments}

A series of computational experiments are conducted to evaluate the effectiveness of the proposed stochastic model. In "Data generation", we itemize the specifics of the instances used in the experiments. In "Computational results on different size instances", we concentrate on evaluating the stochastic model by comparing it with the deterministic model using the expected value of random variables. "Parameter sensitivity analysis" discusses the influence of both the inventory level of medical reserves and designated hospitals' priorities on the different costs. Finally, we perform a cost analysis to identify the factors that cause a more significant total loss in the deterministic problem in the last "Cost analysis".

\section{Data generation}

We generate 15 random instances, including different problem sizes with the number of health care centers ranging from 20 to 200, and the number of scenarios from 20 to 100 . We define the set of designated hospitals as $D$, and the corresponding number is $|D|$. We assume that $|D|$ accounts for $20 \%$ of $|I|$.

The priorities of designated hospitals are the same and are fixed to be $v_{i}=2, i \in D$ and the priorities of other health care centers are set to be $v_{i}=1, i \in I \backslash D$. The allocation cost per unit of supplies (including medical reserves and donated supplies) for each health care center is generated uniformly from $(1,10)$. We simplify the experiments by assuming the unit penalty cost of each health care center is roughly identical and is set as 50, whereas the unit shortage cost is ten times greater than the penalty cost, since shortage may lead to severe consequences during a public health emergency. The lower demand level of each health care center is randomly (uniformly) generated between 0 and 100, while the higher is, between 100 and 200. Both of them are integers. We assume that the inventory level of medical reserves and the amount of donated supplies are related to the problem size, which corresponds to the severity and effects of a public health emergency.

The inventory level of medical reserves $r$ is calculated as follows:

$r=0.5|I| \cdot\left(C_{U B}-C_{L B}\right)$

In addition, the amount of donations under each scenario is drawn uniformly from

$e_{s} \sim U\left[0.025|I| \cdot\left(C_{U B}-C_{L B}\right), 0.1|I| \cdot\left(C_{U B}-C_{L B}\right)\right]$,

where $C_{U B}$ is the upper bound of the integer uniform distribution followed by the higher demand level, and $C_{L B}$ is the lower bound of the integer uniform distribution followed by the lower demand level. They are 200 and 0 , respectively.

Each scenario has the same probability of occurring: $p_{s}=1 /|S|$.

\section{Computational results on different size instances}

In this section, we present and discuss the results of the proposed model, the deterministic model using the expected value of random variables. We have performed the test instances and solved the equivalent linear programming model by the commercial solver Gurobi 9.0.2. All experiments have been conducted on a personal computer with a processor i5 at $2.3 \mathrm{GHz}$ and $8 \mathrm{~GB}$ memory.

According to the construction of the test instance mentioned in "Data generation", we generate 10 instances separately for each data set and report on the expectation and variance of $E V P I_{\text {gap }}$ and $V S S_{\text {gap }}$, and the average solution time of the stochastic program and the deterministic counterpart.

Detailed results for each data set (on average) are listed in Table 1. Columns $|I|$ and $|D|$ provide the number of health care centers and designated hospitals. Column $|S|$ represents the number of scenarios in different sizes. Then, we report the averaged results corresponding to 10 instances, for example, the mean and variance of $V S S_{\text {gap }}$ and $E V P I_{\text {gap }}$, respectively, in the next four columns. The "CPU Time" columns show the average computational time (in milliseconds) required to obtain the optimal solution of 10 independent invocations of two models.

The column "VSS ${ }_{\text {gap }}$ Avg." and the column "EVPI gap Avg." contain the average relative VSS and EVPI in percentage and further details are referred to "Expected value problem and wait-and-see problem". From these columns, we notice that 
Table 1 Computational results of different sizes problem (averages of 10 instances)

\begin{tabular}{|c|c|c|c|c|c|c|c|c|}
\hline \multirow[t]{2}{*}{$|I|$} & \multirow[t]{2}{*}{$|D|$} & \multirow[t]{2}{*}{$|S|$} & \multirow{2}{*}{$\begin{array}{l}V S S_{\text {gap }} \\
\text { Avg. }\end{array}$} & \multirow{2}{*}{$\begin{array}{l}E V P I_{\text {gap }} \\
\text { Avg. }\end{array}$} & \multirow{2}{*}{$\begin{array}{l}V S S_{\text {gap }} \\
\text { Var. }\end{array}$} & \multirow{2}{*}{$\begin{array}{l}E V P I_{\text {gap }} \\
\text { Var. }\end{array}$} & \multicolumn{2}{|c|}{ CPU time $(\mathrm{ms})^{\mathrm{a}}$} \\
\hline & & & & & & & SP & DP \\
\hline \multirow[t]{3}{*}{20} & \multirow[t]{3}{*}{4} & 20 & 4.417 & 3.327 & 0.61 & 1.743 & 7.28 & 0.59 \\
\hline & & 40 & 3.538 & 4.054 & 0.144 & 1.143 & 19.43 & 0.78 \\
\hline & & 100 & 3.392 & 3.38 & 0.164 & 0.288 & 87.54 & 1.42 \\
\hline \multirow[t]{3}{*}{50} & \multirow[t]{3}{*}{10} & 20 & 4.879 & 4.308 & 1.351 & 1.748 & 28.31 & 1.12 \\
\hline & & 40 & 4.215 & 4.257 & 0.525 & 0.857 & 91.35 & 1.56 \\
\hline & & 100 & 3.98 & 5.074 & 0.071 & 0.365 & 222.99 & 3.47 \\
\hline \multirow[t]{3}{*}{100} & \multirow[t]{3}{*}{20} & 20 & 5.301 & 4.437 & 2.427 & 2.503 & 107.18 & 2.36 \\
\hline & & 40 & 4.522 & 4.723 & 0.308 & 0.398 & 385.03 & 3.33 \\
\hline & & 100 & 4.14 & 4.834 & 0.117 & 0.288 & 543.93 & 7.46 \\
\hline \multirow[t]{3}{*}{150} & \multirow[t]{3}{*}{30} & 20 & 6.192 & 3.458 & 2.133 & 1.312 & 228.3 & 3.6 \\
\hline & & 40 & 4.576 & 1.095 & 0.63 & 1.095 & 836 & 6.2 \\
\hline & & 100 & 4.201 & 4.797 & 0.172 & 0.333 & 887.5 & 10.87 \\
\hline \multirow[t]{3}{*}{200} & \multirow[t]{3}{*}{40} & 20 & 5.371 & 3.98 & 1.845 & 0.976 & 347.56 & 4.96 \\
\hline & & 40 & 4.322 & 4.688 & 0.138 & 0.258 & 942.96 & 7.06 \\
\hline & & 100 & 4.198 & 4.817 & 0.168 & 0.401 & 1057.07 & 15.04 \\
\hline
\end{tabular}

${ }^{\text {a }} S P$ stochastic program, $D P$ deterministic program the expectation of $V S S_{\text {gap }}$ ranges from 3.392 to $6.192 \%$, while the expectation of $E V P I_{\text {gap }}$ ranges from 1.095 to $5.074 \%$. The variances of $V S S_{\text {gap }}$ and $E V P I_{\text {gap }}$ reported in columns 6 and 7 show that the differences among all cases are less significant and below 2.503(\%). Analyzing these columns, one can observe that the relative values of VSS and EVPI increase as the number of health care centers increases. This is an indication that an increase in problem size leads to further improvements in the value of both the stochastic solution and the knowledge about the future. Moreover, concerning the limited medical reserves allocation problem, the stochastic program clearly outperforms the deterministic program that uses the expectation of random variables.

As can be observed from Table 1, the average running time, for solving the stochastic programming model of the largestsized instance with 200 health care centers and 100 scenarios, is less than $1.05 \mathrm{~s}$. It indicates that the proposed method enables decision-makers to tackle large-scale allocation problems of limited medical reserves and obtain the optimal integer solution quickly due to its natural unimodularity.

\section{Parameter sensitivity analysis}

In this section, we analyze the impact of two parameters on the total cost (losses): the inventory level of medical reserves and the priority of designated hospitals.

\section{Influence of inventory level on total cost and the first-stage cost}

As mentioned in "Data generation", we generate a mediumsized instance with 50 health care centers and 10 scenarios.
The priorities of designated hospitals and other health care centers are set as $v_{i}=2, i \in D$ and $v_{i}=1, i \in I \backslash D$, respectively. Let the inventory level of medical reserves vary from 0 to 6000 . The objective values of the stochastic program and the expected value problem are obtained by solving the corresponding models. Figure 1 describes the influence of different inventory levels on the stochastic program's objective values and the deterministic program, and we observe that the stochastic program improves more significantly in cost reduction in all cases.

It is evident that the cost for allocating medical reserves in the first stage of both models equals 0 , as the inventory level of medical reserves is 0 . It reflects that both models'

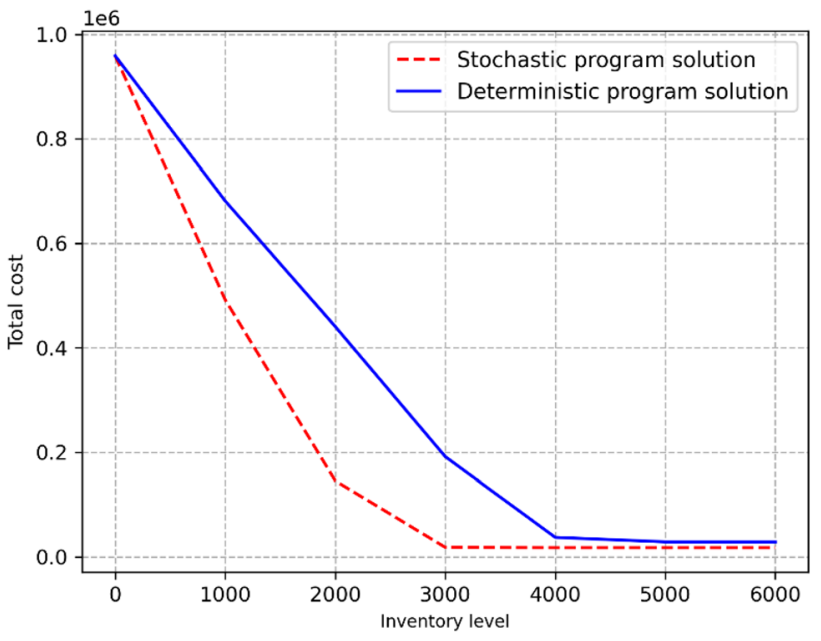

Fig. 1 Influence of different inventory levels on total cost: stochastic program vs. deterministic program 
objective values are similar and equal to recourse cost, which is the sum of costs incurred due to the allocation of donated supplies, shortage, and surplus.

As shown in Fig. 1, the two models' optimal objective values (total cost) maintain a decreasing trend as the inventory level increased, since the shortage cost for unsatisfied demand of all health care centers decreases. In addition, the total cost corresponding to the deterministic program stabilizes when the amount of reserves reaches 4000. By contrast, the total cost of the stochastic program remains steady when it reaches 3000 . We observe that the medical reserves are sufficient, and the inventory level can cover the lower demand levels of all health care centers in this case. The allocation of medical reserves obtained by solving the stochastic model is more reasonable, compared to the allocation from the deterministic model, since excess reserves received by some health care centers result in a higher total cost of the deterministic model, which includes more penalty cost. To summarize, the results suggest the benefit from the stochastic program in the allocation of limited medical reserves whether the amount of reserves is sufficient or not.

Figure 2 displays the influence of different inventory levels on the first-stage allocation cost, indicating that the increase in inventory levels leads to an increase in the number of medical reserves allocated to hospitals in the first stage. In addition, the result obtained by the stochastic program pays more attention on the first-stage allocation, i.e., more medical reserves are employed to cover demand. Compared to the deterministic program, the stochastic program uses limited reserves materials as much as possible to meet demand instead of donated supplies.

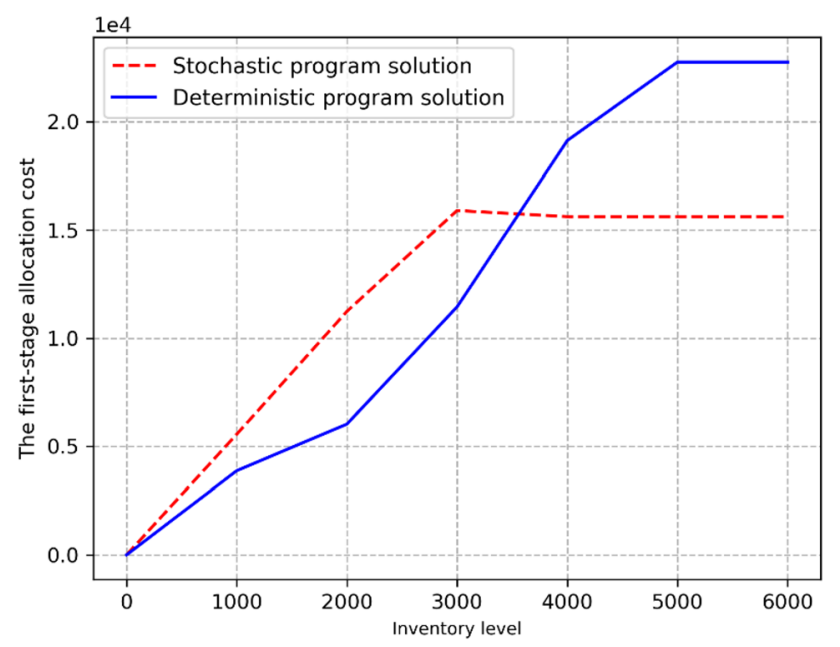

Fig. 2 Influence of different inventory levels on the first-stage allocation cost: stochastic program vs. deterministic program

\section{Influence of priority on total cost and shortage cost of designated hospitals}

We use the same medium-sized instance as described in "Influence of inventory level on total cost and the first-stage cost" and set the inventory level of medical reserves equal to 1000 . Let the priority of designated hospitals vary from 1 to 10 and obtain the stochastic program's optimal objective values and the deterministic program by solving the corresponding models. Figure 3 clearly shows the influence of different priorities of designated hospitals on the total cost. It reveals that using a stochastic program approach for this problem instead of a deterministic approach allows for a considerable improvement both in cost (losses) reduction and in demand fulfill rate of designated hospitals, although with the increase of the priority of designated hospitals, the optimal values of two models also increase. The growth of the stochastic program's optimal value is at a noticeable slower rate than that of the deterministic program. When the priority of designated hospitals is set to be 1 , which means there is no difference between designated hospitals and other health care centers, the unit shortage cost of all health care centers is identical and the total cost in this case is the lowest. However, the optimal value of the stochastic program is still smaller than that of the deterministic program.

Since designated hospitals for severe patients play a key role in the prevention and control of a large-scale public health emergency, their demand should be prioritized. For this reason, we investigate the impact of priority on the shortage cost of designated hospitals, which associates with demand fulfillment. Figure 4 plots different situations by changing the parameter. We observe that two curves show the same pattern: the shortage cost decreases sharply at first and then the rate of increase is slow. For designated

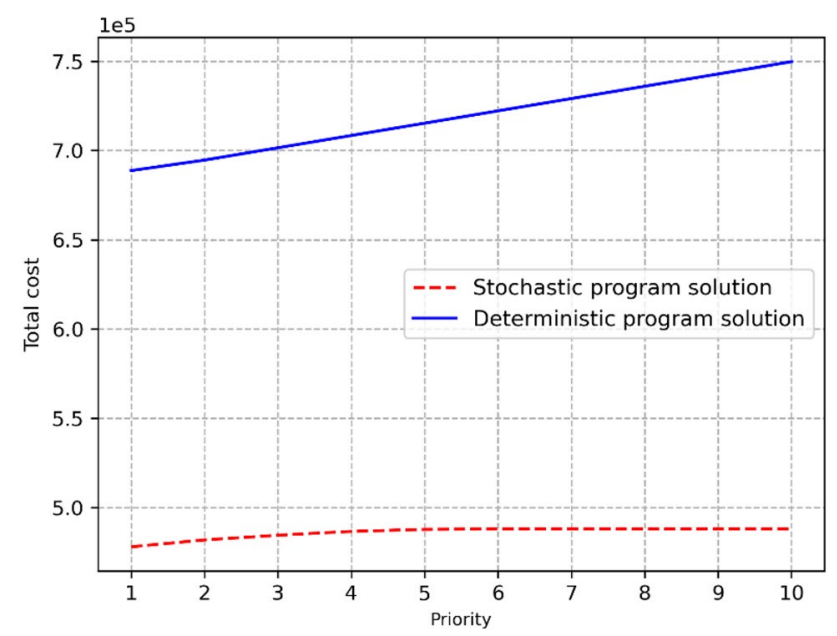

Fig. 3 Influence of different priorities on the total cost: stochastic program vs. deterministic program 


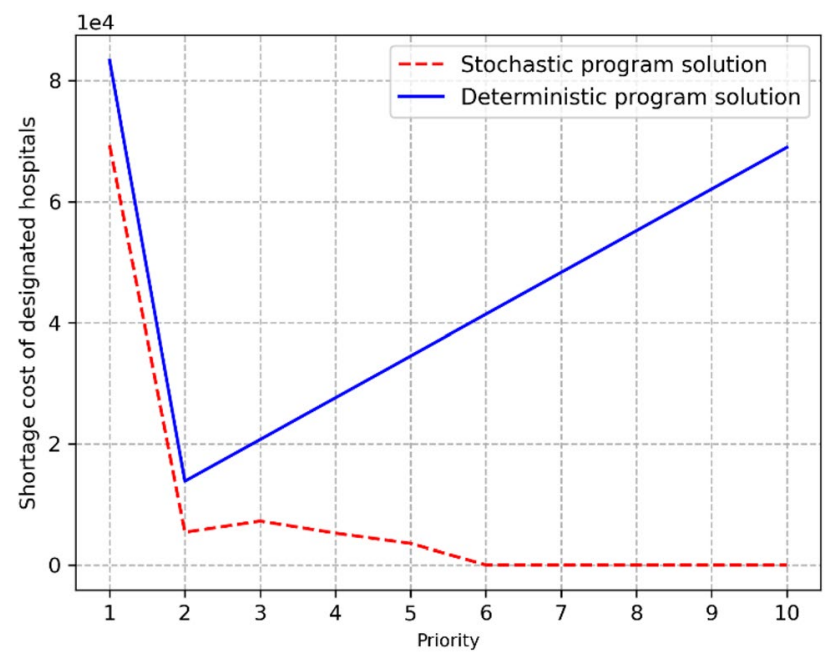

Fig. 4 Influence of different priorities on the shortage cost of designated hospitals: stochastic program vs. deterministic program

hospitals, there is no shortage in the stochastic program, when the corresponding priority of equals to 6 , whereas the shortage cost is still high in the deterministic counterpart. We exploit the parameter to maximize the value of limited reserves by prioritizing the designated hospitals.

\section{Cost analysis}

In this section, we perform a cost analysis to identify the factors that caused more significant losses in the deterministic problem and to further evaluate the effectiveness of the proposed stochastic program. We start setting the inventory level of medical reserves as 3000 and the priority of the designated hospital as 2. Other parameters are generated as described earlier. To obtain the proportion of various costs from stochastic program, deterministic program and waitand-see program, Gurobi is also used to solve these models to optimality. In this medium-sized case, two measures of uncertainty: $V S S_{\text {gap }}$ and $E V P I_{\text {gap }}$ are equal to 11.237 and 0.816 , respectively. Figure 5 illustrates the proportion of various costs from three models. There is no penalty cost when the stochastic program and wait-and-see program are successfully solved to optimality.

Since it is too difficult to predict the relevant information of demand and donations accurately, random variables are often replaced with their expectation to tackle uncertainty by decision-makers, which corresponds to the deterministic program Fig. 5c. As the figure indicates, it is clear that $94.612 \%$ of the total cost comes from shortage cost, and penalty cost takes up approximately $0.041 \%$. It suggests that although shortage costs are incurred after most of the scenarios occur, there still exist

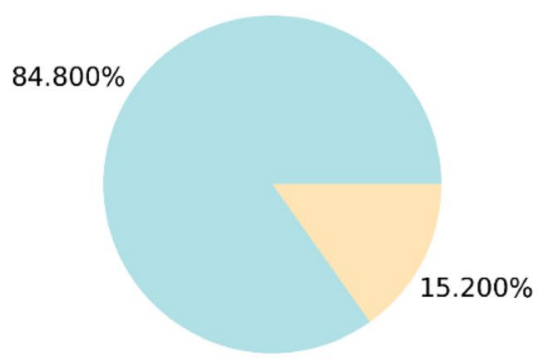

(a) WS Model

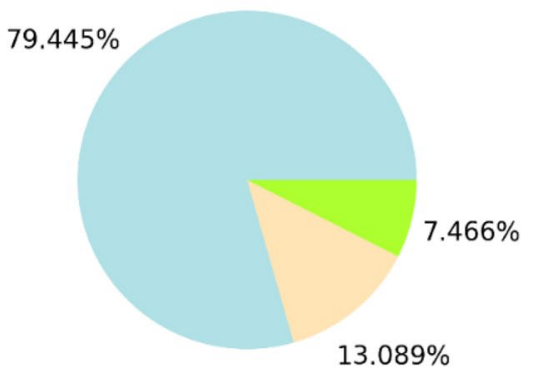

(b) SP Model

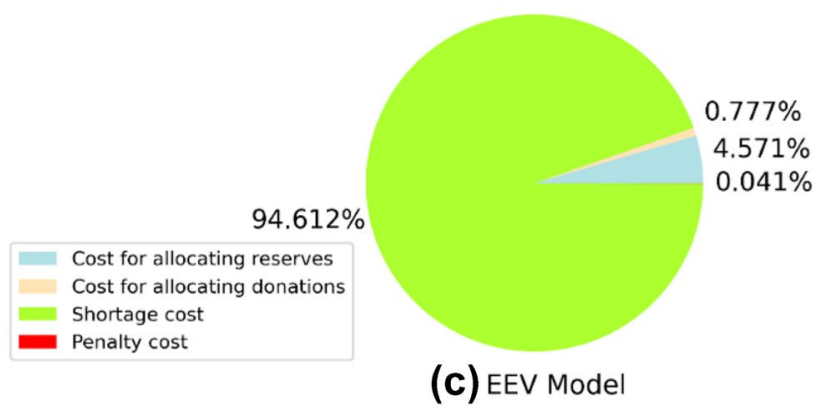

Fig. 5 The proportion of various costs in three models

surplus supplies received by several health care centers under a few scenarios. Consequently, the optimal solution of the deterministic program using the expected value may cause severe losses in a large-scale public health emergency. By contrast, the proposed stochastic program improves more significantly in shortage cost reduction ( $7.466 \%$ vs. $94.612 \%)$, and there is no shortage cost when unknown information (both demand and donations) is revealed (Fig. 5a).

This suggests that we can employ the stochastic programming method to make more reasonable and practical decisions on the allocation problem of limited reserves under uncertainty. In addition, when both reserves and donations cannot cover excess demand, more donations should be appealed for help from the community. In addition, more supportive policies should be implemented to guarantee adequate emergency stockpiles. 


\section{Conclusions}

This study focuses on the allocation of limited medical reserves in the storage pool, when a public health emergency occurs. What is more, accurate information such as the demand of health care centers and the donated supplies is unknown. To obtain the optimal allocation strategy of this research, a two-stage stochastic modeling framework is proposed and its equivalent linear programming model is written. Since the constraint matrix arising in the linear equivalent is totally unimodular, this research can be applied to large-scale integer programs of the allocation of limited medical reserves as well.

The experimental results demonstrate the proposed approach not only benefits the emergency demand fulfill rate but reduces the total losses as well. Compared to the deterministic program that uses the solutions of the expected value problem, the proposed approach results in a significant reduction in terms of losses, by explicitly considering the uncertainty in demand level and donated supplies, as well as the priorities of health care centers.

Future work is needed to handle some limitations in the proposed model. For example, incorporating the inventory level of medical reserves in the allocation problem is worth investigating to obtain the optimal strategy for both reserving and allocating. Various recourse actions to mitigate the uncertainty in demand, such as transshipments mechanism for improving demand fulfill rate, need to be further studied.

\section{Appendix: Proof of Proposition 1}

We denote the constraint matrix of the linear equivalent as $A$, the number of rows of $A$ as $m$. There are sufficient conditions together for $A$ to be totally unimodular:

(1) Every element $a_{i j}$ in $A$ is $-1,0$ or 1 ;

(2) Each column contains at most two nonzero coefficients, i.e., $\sum_{i=1}^{m}\left|a_{i j}\right| \leq 2$;

(3) There exists a partition $\left(M_{1}, M_{2}\right)$ of the set $M$ of rows, where $M_{1}$ comes from the rows of constraints $(12,13$, $14,15)$ after performing row operations make up and $M_{2}$ from constraints (16) to (19), such that each column $j$ containing two nonzero coefficients satisfies $\sum_{i \in M_{1}} a_{i j}-\sum_{i \in M_{2}} a_{i j}=0$.

Therefore, $A$ is totally unimodular.

Funding This research was jointly supported by Beijing Natural Science Foundation under Project No. 9212004 and No. Z180005, the
National Natural Science Foundation of China (NSFC) under Project No. 71771028, and Capital University of Economics and Business Student Academic Newcomer under Project No. 2021XSXR03.

Availability of data and material Not applicable.

Code availability Not applicable.

\section{Declarations}

Conflict of interest On behalf of all the authors, the corresponding author states that there is no conflict of interest.

Open Access This article is licensed under a Creative Commons Attribution 4.0 International License, which permits use, sharing, adaptation, distribution and reproduction in any medium or format, as long as you give appropriate credit to the original author(s) and the source, provide a link to the Creative Commons licence, and indicate if changes were made. The images or other third party material in this article are included in the article's Creative Commons licence, unless indicated otherwise in a credit line to the material. If material is not included in the article's Creative Commons licence and your intended use is not permitted by statutory regulation or exceeds the permitted use, you will need to obtain permission directly from the copyright holder. To view a copy of this licence, visit http://creativecommons.org/licenses/by/4.0/.

\section{References}

1. Manca D, Caldiroli D, Storti E (2020) A simplified math approach to predict ICU beds and mortality rate for hospital emergency planning under Covid-19 pandemic. Comput Chem Eng 140:106945. https://doi.org/10.1016/j.compchemeng.2020. 106945

2. Liu M, Zhang D (2016) A dynamic logistics model for medical resources allocation in an epidemic control with demand forecast updating. J Oper Res Soc 67(6):841-852. https://doi.org/10.1057/ jors.2015.105

3. Barbarosoğlu G, Arda Y (2017) A two-stage stochastic programming framework for transportation planning in disaster response. $\mathrm{J}$ Oper Res Soc 55(1):43-53. https://doi.org/10.1057/palgrave.jors. 2601652

4. Mollah AK, Sadhukhan S, Das P et al (2018) A cost optimization model and solutions for shelter allocation and relief distribution in flood scenario. Int J Disaster Risk Reduct 31:1187-1198. https:// doi.org/10.1016/j.ijdrr.2017.11.018

5. Sun H, Liu J, Han Z et al (2021) Stochastic petri net based modeling of emergency medical rescue processes during earthquakes. J Syst Sci Complex 34(3):1063-1086. https://doi.org/10.1007/ s11424-020-9139-3

6. Zhou QS, Olsen TL (2018) Rotating the medical supplies for emergency response: a simulation based approach. Int J Prod Econ 196:1-11. https://doi.org/10.1016/j.ijpe.2017.11.010

7. Shin K, Lee T (2020) Emergency medical service resource allocation in a mass casualty incident by integrating patient prioritization and hospital selection problems. IISE Transp 52(10):11411155. https://doi.org/10.1080/24725854.2020.1727069

8. Cao H, Huang S (2012) Principles of scarce medical resource allocation in natural disaster relief: a simulation approach. Med Decis Mak 32(3):470-476. https://doi.org/10.1177/0272989X12 437247 
9. Su Z, Zhang G, Liu Y et al (2016) Multiple emergency resource allocation for concurrent incidents in natural disasters. Int J Disaster Risk Reduct 17:199-212. https://doi.org/10.1016/j.ijdrr.2016. 05.003

10. Altay N (2012) Capability-based resource allocation for effective disaster response. IMA J Manag Math 24(2):253-266. https://doi. org/10.1093/imaman/dps001

11. Yu L, Yang H, Miao L et al (2018) Rollout algorithms for resource allocation in humanitarian logistics. IISE Transp 51(8):887-909. https://doi.org/10.1080/24725854.2017.1417655

12. Yu L, Zhang C, Yang H et al (2018) Novel methods for resource allocation in humanitarian logistics considering human suffering. Comput Ind Eng 119:1-20. https://doi.org/10.1016/j.cie.2018.03. 009

13. Yu W, Jia M, Fang X et al (2020) Modeling and analysis of medical resource allocation based on Timed Colored Petri net. Futur Gener Comp Syst 111:368-374. https://doi.org/10.1016/j.future. 2020.05.010

14. Ho TY, Liu S, Zabinsky ZB (2019) A multi-fidelity rollout algorithm for dynamic resource allocation in population disease management. Health Care Manag Sci 22(4):727-755. https://doi.org/ 10.1007/s10729-018-9454-6

15. Lai KK, Cheung MT, Fu Y (2017) Resource allocation in public healthcare: a Team-DEA model. J Syst Sci Complex 31(2):463472. https://doi.org/10.1007/s11424-017-6124-6

16. Kargar B, Pishvaee MS, Jahani H et al (2020) Organ transportation and allocation problem under medical uncertainty: a real case study of liver transplantation. Transp Res Part E 134:101841. https://doi.org/10.1016/j.tre.2020.101841

17. Bastian ND, Ekin T, Kang $\mathrm{H}$ et al (2017) Stochastic multi-objective auto-optimization for resource allocation decision-making in fixed-input health systems. Health Care Manag Sci 20(2):246264. https://doi.org/10.1007/s10729-015-9350-2

18. Huang WT, Chen PS, Liu JJ et al (2018) Dynamic configuration scheduling problem for stochastic medical resources. J Biomed Inform 80:96-105. https://doi.org/10.1016/j.jbi.2018.03.005

19. Chen PS, Lin YJ, Peng NC (2016) A two-stage method to determine the allocation and scheduling of medical staff in uncertain environments. Comput Ind Eng 99:174-188. https://doi.org/10. 1016/j.cie.2016.07.018

20. Zonouzi MN, Kargari M (2020) Modeling uncertainties based on data mining approach in emergency service resource allocation. Comput Ind Eng 145:106485. https://doi.org/10.1016/j.cie.2020. 106485

21. Franco C, Alfonso-Lizarazo E (2020) Optimization under uncertainty of the pharmaceutical supply chain in hospitals. Comput Chem Eng 135:106689. https://doi.org/10.1016/j.compchemeng. 2019.106689
22. Zhang J (2014) Resource planning and allocation problem under uncertain environment. J Syst Sci Complex 28(5):1115-1127. https://doi.org/10.1007/s11424-014-2183-0

23. Hu CL, Liu X, Hua YK (2016) A bi-objective robust model for emergency resource allocation under uncertainty. Int J Prod Res 54(24):7421-7438. https://doi.org/10.1080/00207543.2016.11916 92

24. Boostani A, Jolai F, Bozorgi-Amiri A (2020) Designing a sustainable humanitarian relief logistics model in pre- and postdisaster management. Int J Sustain Transp. https://doi.org/10.1080/15568 318.2020.1773975

25. Yang M, Liu Y, Yang G (2021) Multi-period dynamic distributionally robust pre-positioning of emergency supplies under demand uncertainty. Appl Math Model 89:1433-1458. https://doi.org/10. 1016/j.apm.2020.08.035

26. Gao X (2019) A bi-level stochastic optimization model for multicommodity rebalancing under uncertainty in disaster response. Ann Oper Res. https://doi.org/10.1007/s10479-019-03506-6

27. Zhang X, Liu X, Song X et al (2019) Stochastic location-allocation modelling for emergency mobile blood collection. IFAC-Pap Online 52(13):1114-1119. https://doi.org/10.1016/j.ifacol.2019. 11.345

28. Xiang X, Liu C, Miao L (2017) A bi-objective robust model for berth allocation scheduling under uncertainty. Transp Res Part E 106:294-319. https://doi.org/10.1016/j.tre.2017.07.006

29. Nikzad E, Bashiri M, Oliveira F (2019) Two-stage stochastic programming approach for the medical drug inventory routing problem under uncertainty. Comput Ind Eng 128:358-370. https:// doi.org/10.1016/j.cie.2018.12.055

30. Batista A, Vera J, Pozo D (2020) Multi-objective admission planning problem: a two-stage stochastic approach. Health Care Manag Sci 23(1):51-65. https://doi.org/10.1007/s10729-018-9464-4

31. Feng YY, Wu IC, Chen TL (2017) Stochastic resource allocation in emergency departments with a multi-objective simulation optimization algorithm. Health Care Manag Sci 20(1):55-75. https:// doi.org/10.1007/s10729-015-9335-1

32. Tang J, Wang Y (2015) An adjustable robust optimisation method for elective and emergency surgery capacity allocation with demand uncertainty. Int J Prod Res 53(24):7317-7328. https:// doi.org/10.1080/00207543.2015.1056318

33. Birge JR, Louveaux F (2011) Introduction to stochastic programming. Springer Science and Business Media, Berlin

Publisher's Note Springer Nature remains neutral with regard to jurisdictional claims in published maps and institutional affiliations. 